SOI: $1.1 /$ TAS $\quad$ DOI: $10.15863 /$ TAS

\section{International Scientific Journal} Theoretical \& Applied Science

\author{
p-ISSN: 2308-4944 (print) e-ISSN: 2409-0085 (online) \\ Year: $2016 \quad$ Issue: 2 Volume: 34 \\ Published: 29.02.2016 http://T-Science.org
}

SECTION 7. Mechanics and machine construction.
Sultangaly Z. Zhashen associate Professor of the Department "Transport equipment and technologies" , Ph. D., Taraz state University named after M.Kh. Dulati,

Kazakhstan sultangali54@mail.ru

Sagat Zhunisbekov doctor of technical Sciences, Professor, academician of the National Engineering Academy of the Republic of Kazakhstan, Professor of the Department "Mechanics and engineering",

Taraz state University named after M.Kh. Dulati,

Kazakhstan tar-ti@mail.ru

\title{
EVALUATION OF FATIGUE LIFE OF CRITICAL PARTS, COMPONENTS AND CONSTRUCTION TRUCKS
}

Abstract: A computational-experimental method of evaluating fatigue life of critical parts, components and construction trucks, working in conditions of complex stress state of cyclic and non-stationary loading. In the basis of calculations based on the energy model of high-cycle fatigue. Comparison of results of calculations of fatigue life of critical parts, components and constructions of trucks and available experimental data showed the efficiency of kinetic equations of high-cycle fatigue damage based on energy concept fatigue fracture.

Key words: high Cycle fatigue, durability, energy model high-cycle fatigue, irreversible work of deformation

Language: Russian

Citation: Zhashen SZ, Zhunisbekov S (2016) EVALUATION OF FATIGUE LIFE OF CRITICAL PARTS, COMPONENTS AND CONSTRUCTION TRUCKS. ISJ Theoretical \& Applied Science, 02 (34): 101-107.

Soi: http://s-o-i.org/1.1/TAS-02-34-16 Doi: crostef http://dx.doi.org/10.15863/TAS.2016.02.34.16

\section{УДК 589.385}

\section{ОЦЕНКА УСТАЛОСТНОЙ ДОЛГОВЕЧНОСТИ ОТВЕТСТВЕННЫХ ДЕТАЛЕЙ, ЭЛЕМЕНТОВ И КОНСТРУКЦИИ ГРУЗОВЫХ АВТОМОБИЛЕЙ}

Аннотация: Разработан расчетно-экспериментальный метод оценки усталостной долговечности ответственных деталей, элементов и конструкиии грузовых автомобилей, работающих в условиях сложного ичклического напряженного состояния и нестационарного нагружения. В основу расчетов положена энергетическая модель многочикловой усталости. Сравнение результатов проведенных расчетов усталостной долговечности ответственных деталей, элементов и конструкиии грузовых автомобилей и имеющихся экспериментальных данных указали на эффективность кинетического уравнения многоцикловых усталостных повреждений, основанного на энергетической концепции усталостного разрушения.

Ключевые слова: Многоичиковая усталость, долговечность, энергетическая модель многоичиковой усталости, необратимая работа деформирования.

Как известно, усталостное разрушение один из наиболее распространенных видов разрушения в технике. Если учесть, что разрушение при переменных нагрузках происходит при напряжениях значительно ниже предела прочности, то станет понятной важность вопросов, связанных с изучением природы усталостного разрушения с разработкой средств оценки усталостной прочности материалов. Более правильную оценку усталостной прочности могли бы дать испытания натурных образцов, однако проведение таких опытов очень трудоемко и дорого.

В этой работе сделана попытка разработки расчетно-экспериментального метода оценки усталостной долговечности ответственных деталей, элементов и конструкции грузовых автомобилей, работающих в условиях сложного циклического напряженного состояния и нестационарного нагружения. Экспериментальная часть работы выполнена на установке, описанной в [3, 


\begin{tabular}{|c|c|c|c|c|c|c|}
\hline Impact Factor: & $\begin{array}{l}\text { ISRA (India) } \\
\text { ISI (Dubai, UAB } \\
\text { GIF (Australia) } \\
\text { JIF }\end{array}$ & $\begin{array}{r}=1.344 \\
=0.829 \\
=0.564 \\
=1.500\end{array}$ & $\begin{array}{l}\text { SIS (USA) } \\
\text { PИHЦ (Russia) } \\
\text { ESJI (KZ) } \\
\text { SJIF (Morocco) }\end{array}$ & $\begin{array}{l}=0.912 \\
=0.179 \\
=1.042 \\
=2.031\end{array}$ & $\begin{array}{l}\text { ICV (Poland) } \\
\text { PIF (India) }\end{array}$ & $\begin{array}{l}=6.630 \\
=1.940\end{array}$ \\
\hline
\end{tabular}

5], а в основу расчетов положена энергетическая модель многоцикловой усталости.

Согласно энергетической концепции длительной прочности разрушение наступает в тот момент, когда суммарная необратимая работа деформирования, совершаемая во всех циклах нагружения, достигает некоторого критического значения. Указанная работа определяется суммарной площадью петель гистерезиса. При этом существует ряд экспериментальных методов [6] определения величины энергии, рассеянной за один цикл деформирования при линейном напряженном состоянии.

В табл. 1 приведены известные энергетические критерии, которые могут быть перестроены в кинетические уравнения текущих повреждений. Для этого нужно правую часть каждого из приведенных равенств разделить, на постоянную $\mathrm{C}$ или $\mathrm{S}$ и считать эту величину мерой повреждений, вводя при этом вместо разрушающего - текущее число циклов. Так, например, уравнение Трощенко, помещенное в первой строке табл.1, можно преобразовать к виду

$$
\Pi=\frac{1}{C} \sum_{1}^{N}\left[D-D_{o}\left(\frac{D}{D_{o}}\right)^{\propto}\right]
$$

в дифференциальной форме

$$
\frac{\Delta \Pi}{\Delta N}=\frac{1}{C}\left[D-D_{o}\left(\frac{D}{D_{o}}\right)^{\propto}\right]
$$

Простейшим уравнением такого рода является кинетическое уравнение Фелтнера и Moppoy [7]

$$
\frac{\Delta \Pi}{\Delta N}=F(D),
$$

где $F(D)$ - некоторая функция необратимой работы деформирования в текущем цикле нагружения. Это соотношение равносильно зависимости $F(D)=C$ и учитывает, что накопленные повреждения связаны не со всей, а лишь с некоторой долей полной работы деформирования.

\begin{tabular}{|c|c|c|}
\hline $\begin{array}{l}\text { № } \\
\text { пI/II }\end{array}$ & Энергетические критерии & $\begin{array}{l}\text { Источ } \\
\text { ники }\end{array}$ \\
\hline 1 & $\begin{array}{l}\sum_{1}^{N}\left[D-D_{o}\left(\frac{D}{D_{o}}\right)^{\propto}\right]=\mathrm{C}, \\
\mathrm{D}-\text { энергия, рассеянная в единице объема материала за один цикл и равная площади петли гистерезиса в координатах } \\
\sigma-\varepsilon ; D_{o} \text { - энергия, рассеиваемая за цикл при напряжениях, равньх пределу усталости; } N_{\mathrm{p}} \text { - число циклов до } \\
\text { разрушения; С-константаматериала; } \propto \text {-коэффициент, которыйпринимается равным } 0,5\end{array}$ & [6] \\
\hline 2 & $\begin{array}{l}D_{\Sigma} \text {-суммарная энергия пластическогогистерезиса; } \mathrm{S} \text {-предельная работа } \\
\text { стапического деформирования; } \sigma_{\mathrm{p}} \text { - истинный предел прочности }\end{array}$ & {$[8]$} \\
\hline 3 & $\begin{array}{l}N_{p} \cdot D_{c p}+a=S, \\
a-\text { энергия, соответствующая напряжению } 1 / 2 \quad \sigma_{\text {и }} \text { (истинное напряжение) по диаграмме статического } \\
\text { деформирования, } D_{\text {ср }} \text {-среднеезначениеплощади петлигистерезиса, учитывающеепеременности петли пистерезиса } \\
\text { впроцессе испытания } \quad D_{\text {cp }}=\frac{1}{2} D .\end{array}$ & [9] \\
\hline 4 & $N_{\mathrm{p}}\left(D-D_{o}\right)=D_{\Sigma}^{n}, \quad D_{\Sigma}^{n}$-предельное значение суммарной рассеянной энергии & {$[10]$} \\
\hline 5 & $\begin{array}{l}D_{\sigma}\left(N_{p}-N_{i}\right)=L_{n л}, \\
D_{\sigma}-\text { средняя величина работы разрушения за один цикл нагружения цри напряжении } \sigma ; N_{i}-\text { число } \\
\text { циклов, при котором начинают зарождаться субмикроскопические трещины цри заданном напряжении; } \\
L_{n л} \text { - скрытая теплота плавления }\end{array}$ & {$[11]$} \\
\hline 6 & $\begin{array}{l}\Delta D=\Delta D_{\text {пред}}, \\
\Delta D=k_{1} b\left(\tau_{\text {окm }}+k \sigma_{\text {окm }}\right)^{n} \gamma_{\text {окm}},- \\
\tau_{\text {окm }}, \sigma_{\text {окm }} \text { нормальное и касательное напряжения на октаэдрических площадках; } b-\text { коэффициент, } \\
\text { зависящий от формы петли; } \gamma_{\text {окт }} \text { сдвиговая деформация на октаэдрической шющадке; } \\
k_{1}, k, n \text { - постоянные, связанные со свойствами материала }\end{array}$ & {$[12]$} \\
\hline
\end{tabular}

\section{Известные энергетические критерии.}

Таблица 1

Из рассмотренных критериев (1-5, табл.1) ни один не описывает разрушения при сложном циклическом нагружении. Первой попыткой в этом направлении является критерий 6 (табл 1).

Дальнейшему развитию одного из вариантов энергетической модели разрушения применительно к сложному напряженному состоянию и нестационарному нагружению посвящены работы [1 -4]. В них построено кинетическое уравнение, обобщающее уравнение (3) на сложное циклическое напряженное состояние, причем для определения функциональных и постоянных параметров этого

ISPC Technology and Innovation, 


\begin{tabular}{|c|c|c|c|c|c|c|}
\hline Impact Factor: & $\begin{array}{l}\text { ISRA (India) } \\
\text { ISI (Dubai, UAE } \\
\text { GIF (Australia) } \\
\text { JIF }\end{array}$ & $\begin{array}{l}=1.344 \\
=0.829 \\
=0.564 \\
=1.500\end{array}$ & $\begin{array}{l}\text { SIS (USA) } \\
\text { PИНЦ (Russia) } \\
\text { ESJI (KZ) } \\
\text { SJIF (Morocco) }\end{array}$ & $\begin{array}{l}=0.912 \\
=0.179 \\
=1.042 \\
=2.031\end{array}$ & $\begin{array}{l}\text { ICV (Poland) } \\
\text { PIF (India) }\end{array}$ & $\begin{array}{l}=6.630 \\
=1.940\end{array}$ \\
\hline
\end{tabular}

уравнения требуются лишь данные стандартных опытов на усталость до разрушения при различных циклах растяжения-сжатия.

В указанных работах вводится структурная модель (рис.1), описывающая гипотетический упругопластический материал, обладающий при активном нагружении способностью к линейному упрочнению. Предполагают, что эта модель применима во всем диапазоне напряжений до макроскопического предела текучести и отражает упругие несовершенства материала, причем отпадает необходимость в проведении прецизионных измерений деформаций с целью построения петель гистерезиса.

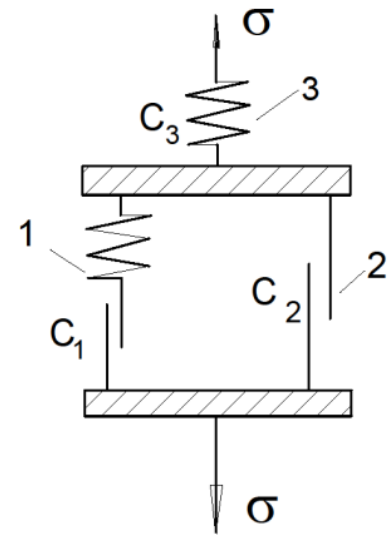

Рисунок 1 - Структурная модель элемента материала [2].

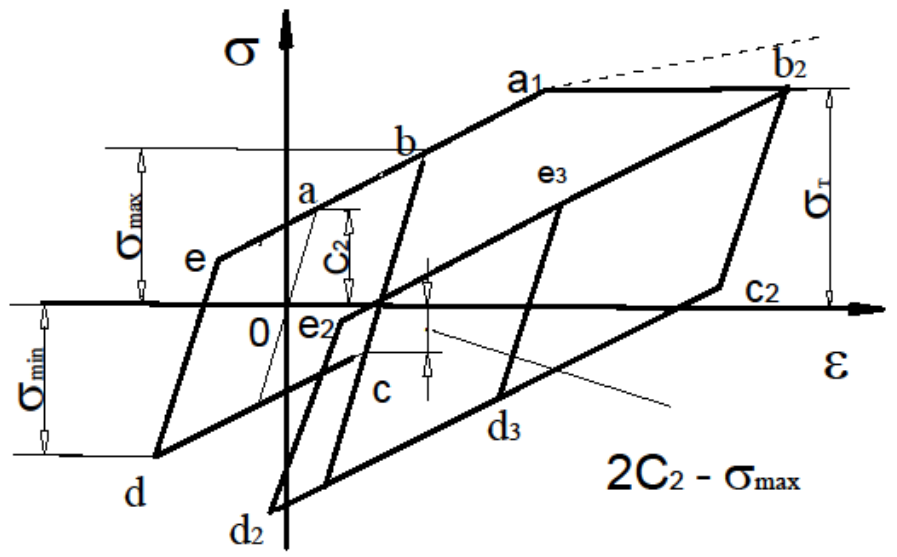

Рисунок 2 - Теоретические диаграммы циклического деформирования [2].

Вводятся следующие параметры структурной модели:

$E_{1}$ - коэффициент жесткости левой ветви I;

$C_{1}$ - предельное сопротивление левой ветви I;

$C_{2}$ - предельное сопротивление цравой ветви 2;

$E_{3}$ - коэффициент жесткости ветви 3.

Диаграмма деформирования элемента рассматриваемого гипотетического материала по модели рис. 1 представлена на рис.2, где линия первичного нагружения материала изображается отрезками прямых $b c-c d-d e-e b$. Нагружение начинается от ненапряженного состояния материала. Тангенс угла наклона линии $о a$ и всех параллельных ей линий равен $E_{3}$, а тангенс угла наклона линии $\mathrm{a}-\mathrm{b}$ и других параллельных ей линий составляет $E_{1} E_{3} / E_{1}+E_{3}$. Максимальное и минимальное за период цикла напряжения ограничены величиной $\sigma_{\max }=C_{1}+C_{2}$. Диаграмма деформирования материала при симметричном цикле нагружения изображается отрезками прямых $b_{2} c_{2}-c_{2} d_{2}-d_{2} e_{2}-e_{2} b_{2}$, a при несимметричном цикле нагружения - отрезками прямых $b_{2} c_{2}-c_{2} d_{3}-d_{3} e_{3}-e_{3} b_{2}$. Смещение этих диаграмм вправо неограничено. Выражение для площади петли пистерезисаполучает ся вследущем виде

$\omega=\frac{2 C_{2}^{2}}{E_{1}}\left[\frac{\sigma_{\max }(1-R)}{C_{2}}-2\right]=\mathrm{A} \aleph\left(\sigma_{\max }, R\right)$,

где $\mathrm{A}$ - некоторая постоянная материала, равная $\frac{2 C_{2}^{2}}{E_{1}} ; \mathrm{R}$ коэффициент асимметриицикла $\mathrm{R}=\sigma_{\min } / \sigma_{\max }$.

Величина площади $\omega$ определяется размахом $\sigma_{\max }(1-R)$ и обрашается в ноль, когда $\sigma_{\max }(1-R) \leq$ 
$2 C_{2}^{2}$. Величина $\omega$ пропорциональна необратимой работе деформирования, некоторая часть которой приводит к накоплениюусталостных повреждений.

Кинетическое уравнение повреждений записывается в виде:

$$
\Pi(N)=\frac{\sigma_{\max }(N)}{C}+\sum_{k=1}^{N} \varphi\left(\aleph_{k}, R_{k}\right)
$$

где $R_{k}$ - текущий коэффициент асимметрии цикла, а текущий параметр $\aleph_{k}$ является безразмерным аргументом некоторой функции поврежденности и определяется из выражения (4):

$$
\aleph_{k}=\omega_{k} \cdot \frac{E_{1}}{2 C_{2}^{2}}=\frac{\omega_{k}}{A}=2\left(\frac{\sigma_{a}^{(K)}}{C_{2}}-1\right) .
$$

Постоянная $C_{2}$ рассматривается, как абсолютный предел выносливости, отождествляемый в литературе с прецизионным цределом текучести для отдельного кристаллического зерна. Эта величина не должна превышать предел ограниченной выносливости материала при симметричном цикле, найденный на такой базе, которая не меньше требуемого ресурса по числу циклов. Значение рекомендуется принимать равным $(0,6 \ldots . .0,8) \sigma_{-1}$. Постоянная С принимается равной истинному сопротивлению разрыву $\sigma_{p}$ и определяется при стандартных испытаниях на статический разрыв цилиндрического образца.

Согласно предварительному условию напряжение $\sigma$ как уже говорилось, не может превосходить значения $\sigma=C_{1}+C_{2}$, поскольку при этом достигается цредельное сопротивление обеих ветвей I и 2. Указанная сумма может рассматриваться как технический предел текучести идеально пластического материала. Вместе с тем рассматриваемую модель можно обобщить и на случай упрочняющегося материала, если представить себе, что параллельно ползунку с сопротивлением $C_{2}$ включена пружина с жесткостью, значительно меньшей $E_{1}$. При этом на рис. 2 вместо горизонтальной линии появится некоторая наклонная линия с возрастающими ординатами, а параллелограмм сдвинется вверх как одно геометрическое целое без изменения его площади. Подобная сдвижка не препятствует проведению нижеописанных расчетов.

В случае регулярного циклического нагружения блоками уравнение (5) преобразуется в следующее:

$$
\Pi=\frac{\sigma_{\max }}{\sigma_{p}}+\sum_{k=1}^{n} \varphi\left(\aleph_{k}, R_{k}\right) \cdot N_{k},
$$

где $\sigma_{\max }$ - максимальное за период цикла растягивающее напряжение в момент определения меры повреждения;

$n$ - количество блоков нагружения до рассматриваемого момента;

א - значение параметра (6) для каждого $k$-го блока, предшествующего $n$-му;

$N_{k}$ - число циклов в $k$-м блоке.

В случае стационарного режима циклического нагружения из уравнения (5) получается такое выражение

$$
\Pi=\frac{\sigma_{\max }}{\sigma_{p}}+\varphi(\aleph, R) \cdot N,
$$

откуда можно определить значение функционального параметра

$$
\varphi(\aleph, R)=\left(1-\frac{\sigma_{\max }}{\sigma_{p}}\right) \frac{1}{N_{p}}
$$

В этом выражении $N_{p}$ - абсцисса усталостной кривой при данном $R$, отвечающая ординате $\sigma_{\max }$. Под усталостной кривой здесь понимается такая, которая отвечает средним долговечностям для каждого уровня напряжений, то есть примерно $50 \quad \%$ вероятности разрушения. Для проведения расчетов при нестационарном циклическом нагружении необходимо располагать серией кривых $\varphi(\aleph, R)$, относящихся к различным коэффициентам асимметрии $R$ (рис. 3). Это требует наличия серии усталостных кривых (рис. 4), относящихся в свою очередь к различным значениям $R=$ const.

При расчете числа циклов до разрушения в заданном режиме нагружения сначала по формуле (9) находится $\aleph$, затем по графикам (рис.3) определяется величина $\varphi(\aleph, R)$, для каждого цикла или блока циклов. После этого должна быть произведена подстановка в правую часть (7), которая приравнивается I:

$$
1=\frac{\sigma_{\max }}{\sigma_{p}}+\sum_{l}^{n} \varphi\left(\aleph_{k}, R_{k}\right) \cdot N_{p,},
$$

откуда и определяется искомая долговечность.

При сложном напряженном состоянии в условиях нарастания интенсивности напряжений упругопластическая деформация гипотетического материала по рис. 1 определяется из выражения

$$
e_{i j}=\frac{1+\vartheta_{3}}{E_{3}} S_{i j}+\frac{3}{2} \cdot \frac{\sigma_{i}-C_{2}}{E_{1}} \cdot \frac{S_{i j}}{\sigma_{i}},
$$

где $e_{i j}$ - компонента девиатора деформаций;

$S_{i j}$ - компонента девиатора полных напряжений $\left(S_{i j}=S_{i j}{ }^{(3)}\right)$, где цифра 3 указывает третье звено по рис. 1;

$\sigma_{i}$ - интенсивность напряжений;

$\vartheta_{3}$ - коэффициент поперечной деформации;

$E_{3}$ - модуль упругости третьего звена.

При сложном циклическом нагружении модель (рис.1) действует следующим образом. Исходным состоянием материала считается такое состояние, в котором начальные и внешние напряжения равны нулю. Предположим, что в некоторый момент времени интенсивность напряжений начинает нарастать. Сначала пока $\sigma_{i}<C_{2}$ деформируется только элемент 3 , при этом упругопластические деформации отсутствуют. Начиная с момента достижения интенсивностью напряжений значения $\quad \sigma_{i}=C_{2}$ развиваются упругопластические деформации, определяемые по формуле (11). Наконец после достижения через определенный промежуток времени своего максимального значения интенсивность $\sigma_{i}$ начинает уменышаться. 


\begin{tabular}{l|lrl|l|ll} 
& ISRA (India) & $=\mathbf{1 . 3 4 4}$ & SIS (USA) & $=\mathbf{0 . 9 1 2}$ & ICV (Poland) & $=\mathbf{6 . 6 3 0}$ \\
Impact Factor: & ISI (Dubai, UAE) $=\mathbf{0 . 8 2 9}$ & PUHU (Russia) $=\mathbf{0 . 1 7 9}$ & PIF (India) & $=\mathbf{1 . 9 4 0}$ \\
& GIF (Australia) & $\mathbf{0 . 5 6 4}$ & ESJI (KZ) & $=\mathbf{1 . 0 4 2}$ & & \\
& JIF & $=\mathbf{1 . 5 0 0}$ & SJIF (Morocco) $=\mathbf{2 . 0 3 1}$ & &
\end{tabular}

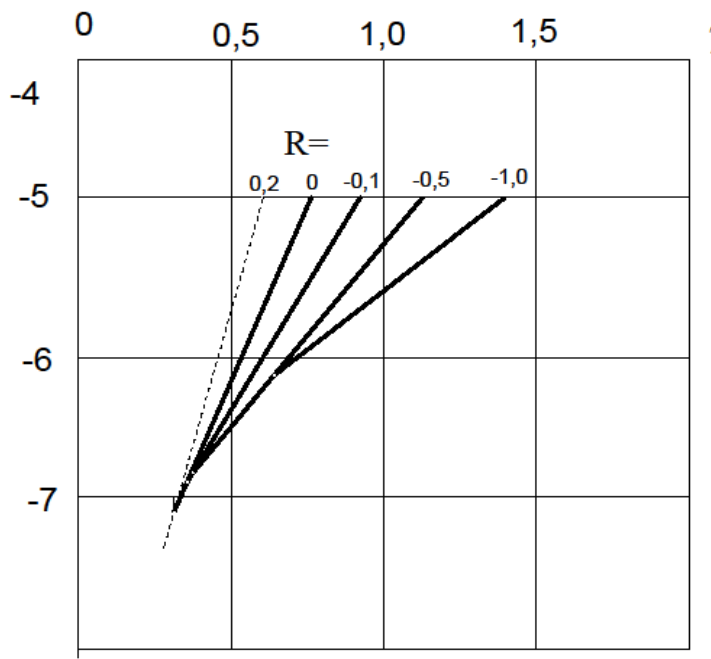

$\lg \varphi(\chi, R)$

Рисунок 3 - Функция удельной работы деформирования для стали 45 по [2].

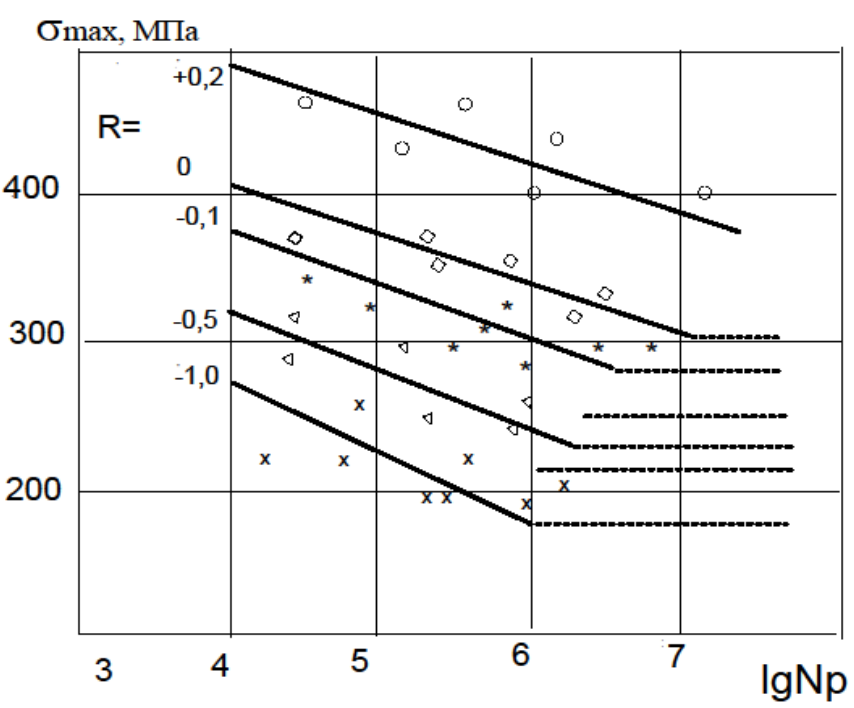

Рисунок 4 - Кривые усталости для стали 45 по [2].

В этих условиях разгрузки элемент 2 становится абсолютно жестким, то есть его деформация прекращается, одновременно прекращается деформация элемента I и напряжения в этом элементе сохраняют те самые значения, которые они имели до начала разгрузки. Деформация элемента 3 уменьшается, так же как и напряжения в элементе 2, которые могут при определенных условиях достигать нуля, перейти через ноль и снова возрастать по любому закону.

Для расчета деформаций в условиях сложного циклического нагружения в работах [1-4] предлагается следующая методика. Пусть интенсивность напряжения возрастает, начиная от нулевого значения, до тех пор, пока $\sigma_{i}<C_{2}$, деформируется, как уже указывалось только элемент 3. С переходом через состояние $\sigma_{i}=C_{2}$ возникают упругопластические деформации, которые вычисляются по формуле (11). Затем интенсивность $\sigma_{i}$ достигает максимального значения и начинает падать. В этом состоянии проводится мысленная разгрузка всей системы на величину интенсивности напряжений $\sigma_{i}^{(2)}=C_{2}$ причем с каждой компоненты полного девиатора напряжений снимается в алгебраическом смысле величина

В этом состоянии

$$
S_{i j}{ }^{(2)}=\left(S_{i j}-S_{i j}{ }^{(I)}\right)=S_{i j}-\frac{3}{2} E_{l} \cdot e_{i j}{ }^{(2)}=S_{i j}\left(1-\frac{\sigma_{i}-C_{2}}{\sigma_{i}}\right) .
$$




\begin{tabular}{|c|c|c|c|c|c|c|}
\hline Impact Factor: & $\begin{array}{l}\text { ISRA (India) } \\
\text { ISI (Dubai, UAE } \\
\text { GIF (Australia) } \\
\text { JIF }\end{array}$ & $\begin{array}{l}=1.344 \\
=0.829 \\
=0.564 \\
=1.500\end{array}$ & $\begin{array}{l}\text { SIS (USA) } \\
\text { PИНЦ (Russia) } \\
\text { ESJI (KZ) } \\
\text { SJIF (Morocco) }\end{array}$ & $\begin{array}{l}=0.912 \\
=0.179 \\
=1.042 \\
=2.031\end{array}$ & $\begin{array}{l}\text { ICV (Poland) } \\
\text { PIF (India) }\end{array}$ & $\begin{array}{l}=6.630 \\
=1.940\end{array}$ \\
\hline
\end{tabular}

$$
S_{i j}=S_{i j}{ }^{\left({ }^{3}\right)}=S_{i j}{ }^{(l)}=\frac{\sigma_{i}-C_{2}}{\sigma_{i}} S_{i j} .
$$

Отсчет деформаций, возникающих в процессе дальнейшего нагружения, ведется от рассматриваемого состояния разгрузки, причем расчетная формула (11) остается в силе вплоть до момента следующей разгрузки, если в эту формулу вместо $S_{i j}$ вводятся приращения $\Delta S_{i j}$, отсчитываемые от указанного состояния разгрузки, причем вторичная пластическая деформация элемента 2 появится при условии $\Delta \sigma_{i}=$ $C_{2}$. В итоге расчета строятся графики зависимости каждой компоненты девиатора $S_{i j}$ от соответствующей компоненты девиатора пластических деформаций $e_{i j}{ }^{(2)}$, на которых при соответствующих условиях получаются петли пластического гистерезиса. Сумма таких петель за один цикл нагружения дает работу необратимой деформации.

В случае регулярного циклического нагружения, когда отдельные компоненты напряжений изменяются по разным циклам, вводится понятие некоторого приведенного коэффициента асимметрии цикла, определяемого по выражению

$$
\Omega_{\text {пр }}=\frac{\sum \Omega_{\mathrm{ij}} \cdot \omega\left(\cdot \sigma_{i j}\right)}{\omega_{\Sigma}},
$$

где $\omega\left(\cdot \sigma_{i j}\right)$ - площадь петли гистерезиса, образующаяся за счет указанной компоненты тензора напряжений;

$\omega_{\Sigma}$ - суммарная площадь петель гистерезиса в одном цикле нагружения,

$$
\Omega=\frac{\sigma_{m}}{\beta \sigma_{m}+\sigma_{a}}=\frac{1+R}{\beta(I+R)+(I-R)}=\frac{1+R}{\beta+1+(\beta-I) R} .
$$

Величина $\beta$ подбирается каждый раз таким образом, чтобы параметр $\Omega$ ни при одном из встречающихся режимов изменения компонентов напряжения не обращался в бесконечность. В частном случае, как ни один из коэффициентов асимметрии не обращается в бесконечность, можно пользоваться формулой

$$
\mathrm{R}_{\text {пр }}=\frac{\sum \mathrm{R}_{\mathrm{ij}} \cdot \omega\left(\cdot \sigma_{i j}\right)}{\omega_{\Sigma}},
$$

где $\mathrm{R}_{\mathrm{ij}}$ - коэффициенты асимметрии цикла соответствующей компоненты тензора напряжений $\sigma_{i j}$.

B paботе [4] описаны проведенные испьтания на усталость в условиях плоского циклического напряженного состояния образцов стали 10 и 45 при различньх режимах стационарного и нестационарного нагружения. В некоторых случаях режим изменения осевого напряжения в образцебьл бигармоническим при изменении оқружного напряжения по синусоидальному отнулевому циклу. Полученные экспериментальные данные использованы для проверки условий эквивалентности различньх циклических напряженньх состояний по ряду известньх силовых гритериев усталостного разрушения. Проведенный расчет для нестационарных режимов циклического нагружения указал на приемлемость формулы линейного суммирования повреждений при условии, что разрушающее число циклов для режима сложного циклического нагружения в каждом блоке известно заранее.

Сравнение результатов проведенных расчетов усталостной долповечности ответственных деталей, элементов и конструкции грузовых автомобилей и имеющихся экспериментальных данных указали на эффективность кинетического уравнения многоцикловых усталостньх повреждений, основанного на энергетической концепции усталостного разрушения. Об этом можно судить на основе сравнения экспериментальных чисел циклов до разрушения и расчепньх чисел $N_{\mathrm{p}}[3,4]$.

\section{References:}

1. Pavlov PA, Kondakova ON, Konev AI (1981) Razvitie energeticheskoy modeli mnogotsiklovogo ustalostnogo razrusheniya. - / Tez.dokl., Perm': Sektsiya ustalosti nauchnogo soveta GKNT, 1981, pp.61-62.

2. Zhashenov SD (1982) Energeticheskie modeli mnogotsiklovoy ustalosti konstruktsionnykh metallov. V sb.: Voprosy prochnosti elementov sel'skokhozyaystvennykh, stroitel'nykh i meliorativnykh mashin. TIIIMSKh, 1982, vyp. 124.

3. Zhashenov SD (1983) Eksperimental'noe issledovanie mnogotsiklovoy ustalosti konstruktsionnykh staley pri slozhnom nestatsionarnom nagruzhenii $\mathrm{i}$ razvitie energeticheskogo metoda rascheta povrezhdeniy: Avtoref. dis. ... kand. tekhn. nauk. - L., 1983. - 16 p.

4. Zhashen SZ (2004) O predelakh vozmozhnosti primeneniya energeticheskoy modeli mnogotsiklovoy ustalosti pri slozhnom mnogokomponentnom nagruzhenii. Nauka i obrazovaniya Yuzhnogo Kazakhstana. Respublikanskiy nauchnyy zhurnal №2(37) 2004.

5. Pavlov PA, Sherstnev VA, Kasymov UT, Zhashenov SD (1982) Gidropul'satsionnaya mashina dvukhstoronnego deystviya. - Inform. listok, LenTsNTI, L., 1982, № 708-82. 


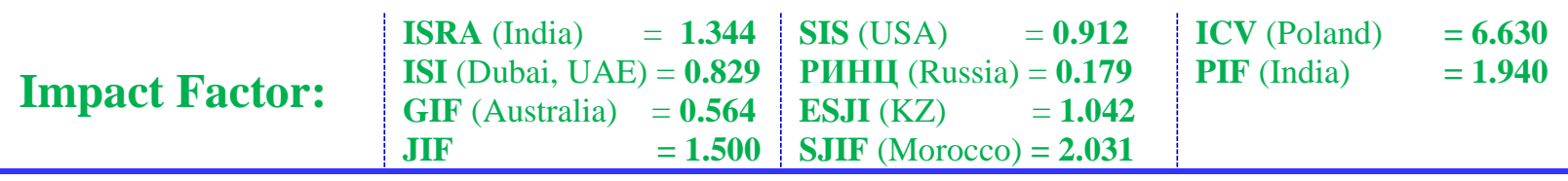

6. Troshchenko VT (1981) Deformirovanie i razrushenie metallov pri mnogotsiklovom nagruzhenii. - Kiev: Naukova dumka, 1981. $344 \mathrm{p}$.

7. Feltner CE, Morrow ID (1961) Microplastic strain hysteresis energy asa criterion for fatigue. - I.Basic Eng. D, 1961, 83, № 1, p. 15-21.

8. Thiruvengadam A (1966) High frequency fatigue of metals and their cavitation - damage resistance. - Trans. ASME, 1966, № 3, p. 105112.

9. Stowell E (1966) A study of the energy criterion for fatigue. - Nucl. Eng. And Des., 1966, № 3, pp. 32-40.
10. Chang CS, Pimbley WT, Cunway HD (1968) An analysis of metal fatigue based on hysteresis energy. Exp. Mech., 1968, 8, № 3, pp. 41.

11. Ivanova VS (1962) Strukturno energeticheskaya teoriya ustalosti metallov. - Moscow: 1962, pp. 11-23.

12. Gol'tsev DI (1953) Ob usloviyakh prochnosti pri peremennykh nagruzkakh i slozhnom napryazhennom sostoyanii. V kn. : Voprosy dinamicheskoy prochnosti. Riga, 1953. pp. 3439. 